Proceedings of the 30th Polish Seminar on Positron Annihilation, Jarnottówek 1998

\title{
POSITRONIUM IN SMALL AND LARGE VOIDS IN VYCOR GLASS
}

\author{
K. Ciesielski ${ }^{a}$, A.L. DaWidowicz ${ }^{b}$ AND T. GoworeK ${ }^{a}$ \\ anstitute of Physics, Maria Curie-Skłodowska University \\ Pl. M. Curie-Skłodowskiej 1, 20-031 Lublin, Poland \\ ${ }^{b}$ Department of Chemical Physics, Maria Curie-Skłodowska University \\ Pl. M. Curie-Skłodowskiej 3, 20-031 Lublin, Poland
}

The intensities of ortho-positronium components related to the pores and to small voids in the bulk were determined for a series of Vycor glasses. The ratio of "pore fraction" to the total o-Ps yield depends on the specific surface area of the porous medium. The diffusion coefficient for $o$-Ps was found equal to $3.7 \times 10^{-5} \mathrm{~cm}^{2} / \mathrm{s}$.

PACS numbers: $78.70 . \mathrm{Bj}$

\section{Introduction}

Positronium (Ps), the bound state of electron and positron, locates in the regions of low electron density (voids) in the medium structure [1]. The ortho-Ps lifetime, which in vacuum amounts $\approx 140 \mathrm{~ns}$, in the voids reduces due to the interaction of the positron with surrounding molecular electrons (the pick-off process). In porous materials, like silica gels and Vycor glasses, there are two distinctly different types of voids:

- the pores, i.e. complex channel-like structures with average diameter usually over $4 \mathrm{~nm}$,

- small, subnanometer free spaces in the amorphous structure of the bulk, with the sizes not exceeding $0.4-0.6 \mathrm{~nm}$.

The o-Ps lifetimes in typical pores are larger than $40 \mathrm{~ns}$, while in small voids they are 2 ns or less, thus distinction of these two groups of free spaces is easy. This paper represents an attempt to study the distribution of Ps into two groups of voids as a function of specific pore surface area.

\section{Experimental}

Positron source ${ }^{22} \mathrm{Na}$ in a Kapton envelope was placed between two layers of Vycor glass (grains $35-75 \mu \mathrm{m}$ ) and pressed together in a brass container. The container was fixed to the cold finger inside the chamber evacuated to $\approx 0.4 \mathrm{~Pa}$; this pressure is sufficient to eliminate Ps quenching by the oxygen in the air. The other end of finger could be submerged in liquid nitrogen or connected to the heater. The temperature controlling heater was placed between the sample container and the finger. The temperature of container was kept constant with the accuracy $\pm 0.2 \mathrm{~K}$ ( $\pm 0.5 \mathrm{~K}$ at highest temperatures). The parameters of glass samples are collected in Table. 
TABLE

Sample characteristics. The errors indicated in the columns 4, 5 are fitting errors only.

\begin{tabular}{c|c|c|c|c|c}
\hline \hline Sample & $\begin{array}{c}\text { Specific } \\
\text { surface area } \\
(S)\left[\mathrm{m}^{2} / \mathrm{g}\right]\end{array}$ & $\begin{array}{c}\text { Pore volume } \\
\left(V_{\mathrm{p}}\right) \\
{\left[\mathrm{cm}^{3} / \mathrm{g}\right]}\end{array}$ & $\begin{array}{c}\text { Total } 0 \text {-Ps } \\
\text { intensity } \\
\left(I_{2}+I_{3}\right) \\
{[\%]}\end{array}$ & $\begin{array}{c}\text { Intensity of } \\
\text { o-Ps decaying } \\
\text { in the pores } \\
\left(I_{3}\right)[\%]\end{array}$ & $\kappa$ \\
\hline 1 & 12.5 & 0.99 & $42.9 \pm 0.2$ & $3.8 \pm 0.1$ & 0.09 \\
2 & 17.3 & 1.02 & $36.4 \pm 0.2$ & $4.0 \pm 0.1$ & 0.11 \\
3 & 37.0 & 0.95 & $32.7 \pm 0.1$ & $6.9 \pm 0.1$ & 0.21 \\
4 & 64.1 & 0.90 & $35.0 \pm 0.3$ & $15.7 \pm 0.1$ & 0.45 \\
5 & 65.2 & 0.92 & $37.7 \pm 0.3$ & $14.7 \pm 0.1$ & 0.39 \\
6 & 87.1 & 0.88 & $28.1 \pm 0.3$ & $16.0 \pm 0.1$ & 0.57 \\
7 & 165.6 & 0.99 & $32.2 \pm 0.2$ & $26.0 \pm 0.1$ & 0.81 \\
8 & 187.1 & 0.87 & $42.3 \pm 0.1$ & $34.7 \pm 0.1$ & 0.82 \\
9 & 184.8 & 0.54 & $35.2 \pm 0.2$ & $33.0 \pm 0.2$ & 0.94 \\
10 & 305.4 & 0.46 & $36.5 \pm 0.1$ & $31.1 \pm 0.1$ & 0.85 \\
11 & 351.2 & 0.46 & $32.8 \pm 0.1$ & $29.0 \pm 0.1$ & 0.88
\end{tabular}

The lifetime spectra were measured with a standard fast-slow spectrometer; the energy window in the stop counter was widely open $(80 \%$ of the energy range) to accept the largest possible part of the continuous energy spectrum of three-quantum annihilation. The time base was $2 \mu$ s (channel definition $256 \mathrm{ps}$ ); the resolution of spectrometer was $\approx 300 \mathrm{ps}$; typical statistics was $2 \times 10^{6}$ coincidences per spectrum. The spectra were processed by LT program [2].

\section{Results and discussion}

The shortest-lived component represents the annihilation of free positrons and of singlet para-Ps. At our channel definition they cannot be resolved and are seen as one, with $\tau_{1} \approx 400 \mathrm{ps}$. The component related to ortho-Ps in the bulk has the lifetime $\tau_{2}$ of about $1.45 \mathrm{~ns}$; it means that, according to the spherical model [3], o-Ps is located in the free spaces $0.23 \mathrm{~nm}$ in radius. The longest-lived component is not purely exponential; it is distorted in the initial part by the annihilation of nonthermalized $o-\mathrm{Ps}$ [4]. One can approximate it by two discrete components or by one component with continuous $\lambda$ distribution. In this paper that other variant was chosen. The pore radii of all investigated glasses were over $4 \mathrm{~nm}$, thus the longest-lived component (if one neglects the initial range of delays) in all samples reached, or was close to, the saturation value $\tau_{3} \approx 140 \mathrm{~ns}$, like for $o$-Ps in vacuum [5]. The sum of intensities of both o-Ps components $I_{2}+I_{3}$ varies in relatively narrow limits $(30-40) \%$, but the ratio of these intensities can change from domination of the short-lived one to its reduction to several percent. The $I_{3}$ component contains almost entirely the three-quantum decays, while $I_{2}$ 


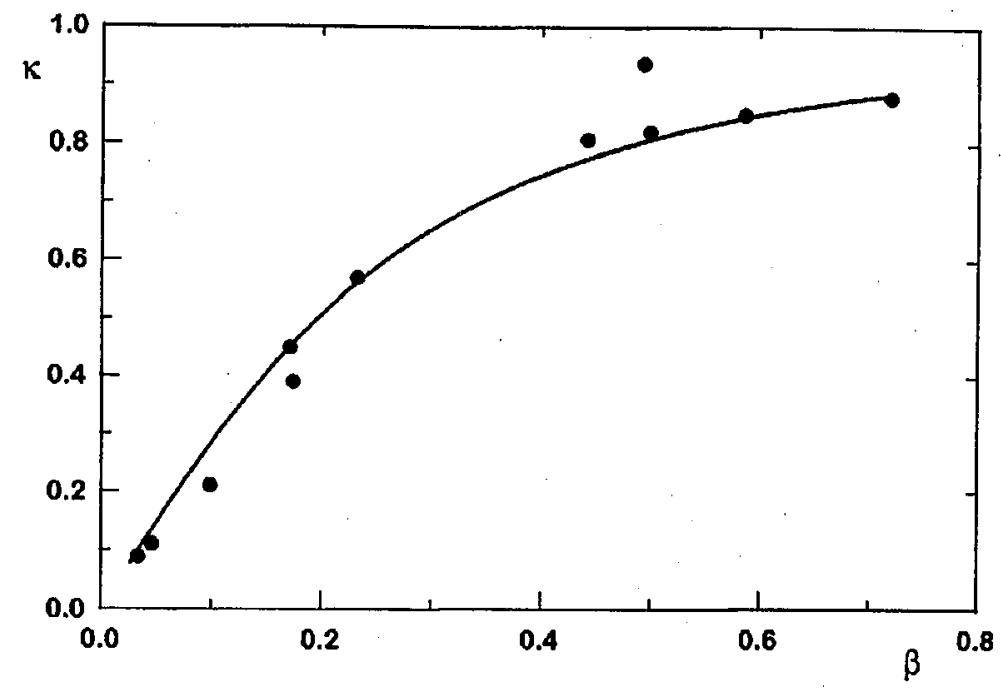

Fig. 1. Relative intensity of $o$-Ps in the pores $(\kappa)$ vs. $\beta$ parameter. The solid line represents the fitting of Eq. (1) to the experimental points.

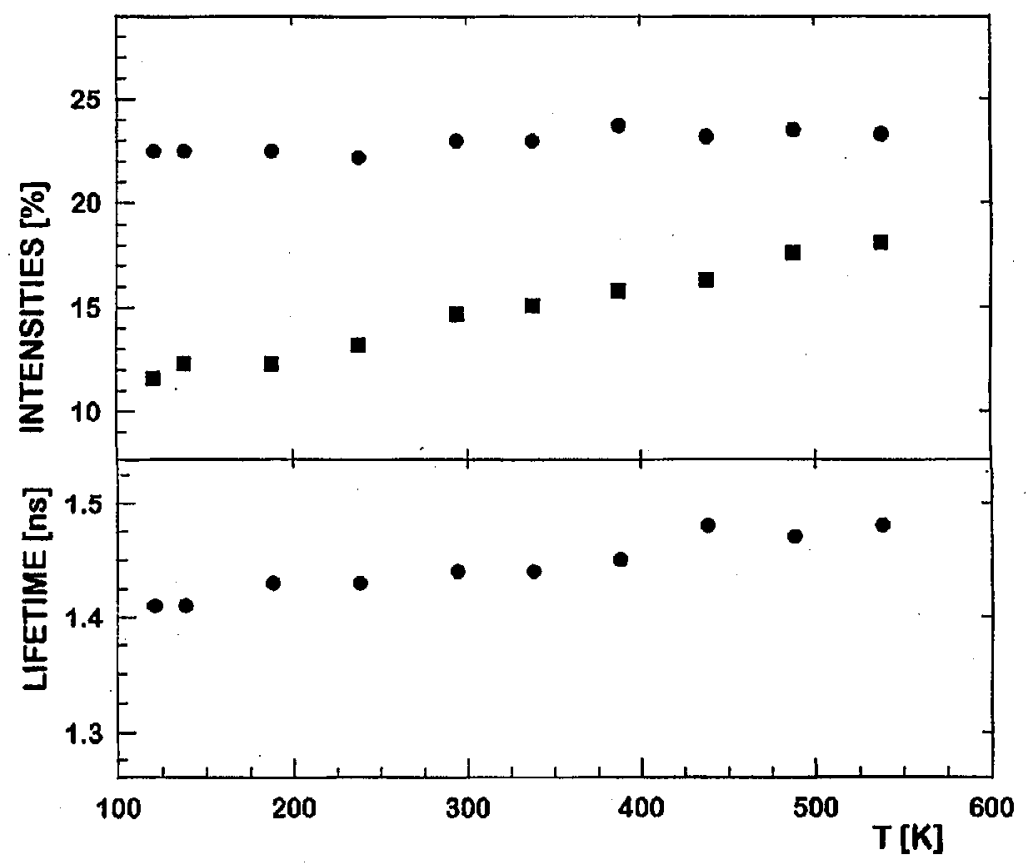

Fig. 2. Temperature dependence of the bulk o-Ps lifetime $t_{2}$ and of the intensities $I_{2}$ (points), $I_{3}$ (squares) for glass sample No. 5 (see Table). Fitting errors are comparable to the size of symbols. 
represents two-quantum events. The difference in efficiency of the registration of . two- and three-quantum annihilations can distort the value of $I_{3}$ intensity, however the possible distortion is the same for all samples.

The intensity of the $140 \mathrm{~ns}$ component is determined by the pore surface area. The small voids in the bulk appear in large concentration, thus only these $o$-Ps atoms which were formed in a thin layer near the surface have the chance to diffuse to the pores. Figure 1 shows the yield of $o$-Ps trapped in the pores as a function of specific pore area. When this area is $17 \mathrm{~m}^{2} / \mathrm{g}$ the pore component is $3.8 \%$ only, while the bulk component is $39 \%$, the proportion is reversed at $200 \mathrm{~m}^{2} / \mathrm{g}$. The distribution of $o$-Ps into pores and bulk voids can be described by the model proposed by Brandt and Paulin [6] for fine powders and modified by Venkateswaran et al. [7] to be applicable to porous media. According to this model the ratio $\kappa=I_{3} /\left(I_{2}+I_{3}\right)$ is

$$
\kappa=\frac{3}{2} \beta\left[1-\beta^{2}+(1+\beta)^{2} \exp (-2 / \beta)\right],
$$

where $\beta=S \rho\left(D \tau_{2}\right)^{1 / 2} / 3, S$ is the surface area, $\rho$ is bulk glass density, and $D$ is the diffusion coefficient. The solid curve in Fig. 1 shows $\kappa$ fitted to the experimental data. The diffusion coefficient for Ps in the Vycor glass was found equal to $D=3.7 \times 10^{-5} \mathrm{~cm}^{2} / \mathrm{s}$. It is slightly larger than that determined by Venkateswaran et al. [7] for porous polymers (amberlites), and smaller than that found by Brandt and Paulin for pure fine powdered silica.

The temperature dependence of lifetime spectrum was investigated for the sample No. 5 in which the intensities $I_{2}$ and $I_{3}$ are comparable. The result is shown in Fig. 2. The lifetime $\tau_{2}$ is almost constant, the rise of the temperature by $420 \mathrm{~K}$ changes it by $4.8 \%$ only. The ratio of intensities $\kappa$ rises with temperature too, however the plot $\ln \left(I_{2} / I_{3}\right)$ vs. $1 / T$ is not linear as one can expect from the diffusion model.

\section{References}

[1] O.E. Mogensen, Positron Annihilation in Chemistry, Springer Verlag, Berlin 1995.

[2] J. Kansy, Nucl. Instrum. Methods Phys. Res. A 374, 235 (1996).

[3] M. Eldrup, D. Lightbody, J.N. Sherwood, Chem. Phys. 63, 51 (1981).

[4] C. Dauwe, G. Consolati, T. van Hoecke, D. Seghers, Nucl. Instrum. Methods Phys. Res. A 371, 497 (1996).

[5] K. Ciesielski, A.L. Dawidowicz; T. Goworek, B. Jasińska, J. Wawryszczuk, Chem. Phys. Lett. 289, 41 (1998).

[6] W. Brandt, R. Paulin, Phys. Rev. Lett. 21, 193 (1968).

[7] K. Venkateswaran, K.L. Cheng, Y.C. Jean, J. Phys. Chem. 88, 2465 (1984). 